\title{
The Inflammatory Cytokine IL-21 is Expressed by Splenic Neutrophils in Response to Transplantation of Allogeneic Cells
}

\author{
John S. Thompson ${ }^{1,2 *}$, Debra L. Hardin ${ }^{2}$, Judy F. Glass ${ }^{1}$, Joshua Dziba ${ }^{1}$, Jeffrey Campion ${ }^{3}$ and \\ Stephen A. Brown ${ }^{1,2}$ \\ ${ }^{1}$ VA Medical Center, Lexington Kentucky 40502 \\ ${ }^{2}$ Department of Internal Medicine, College of Medicine, Lexington, Kentucky 40536 \\ ${ }^{3}$ College of Pharmacy Lexington Kentucky 40536
}

Received: May 20, 2016; Accepted: June 03, 2016; Published: June 08, 2016

*Corresponding author: John S. Thompson, VA Medical Center 1101 Veterans Drive, Lexington KY 40502 and Department of Internal Medicine, University of Kentucky 800 Rose Street Lexington KY 40536, Tel: 859-233-4511 x 4580; Cell: 859-539-1614; Fax: 859-281-4989; E-mail: jsthom1@uky.edu

\begin{abstract}
Absract
We have previously reported that GR-1 neutrophil/monocytes rose dramatically in the spleen, peaked by day 7 and declined through day 14. This period corresponded to the peak of acute Graft-VersusHost Disease (aGVHD) in BALB/c mice transplanted with allogeneic donor cells. We now asked: what cytokines did these splenic neutrophil/monocytes express on day 7 and 14 post transplant?
\end{abstract}

$\mathrm{BALB} / \mathrm{c}$ mice were transplanted with allogeneic B6 or syngeneic BALB/c donor cells. Long term survival was recorded through day 31. Other groups were sacrificed on days $3,5,7,14,21$ and 31 days post transplant to record the total number of cells in the spleens and their phenotypes. Neutrophils were isolated from the spleens of mice transplanted with B6 and BALB/c cells on days 7 and 14 .

Daily body weight demonstrated a transient drop in the syngeneic transplants on day 2 but a much greater drop with its nadir at day 7 and never fully recovering through 31 days. CD8/CD4 T lymphocytes peaked in the spleen on day 5 and were followed on day 7 by GR-I cells in all of the allogeneic transplants. In syngeneic transplants this early rise in lymphocytes did not occur and GR-1 cells peaked on day 14. Highly purified neutrophils were isolated in two separate experiments from the spleens on days 7 and 14 post transplant. In both experiments day 7 allogeneic neutrophils expressed significantly elevated levels of Interleukin -21 (IL-21) mRNA whereas the day 7 and 14 syngeneic cells expressed lower but significant levels of TNF $\alpha$ Intracellular IL-21 was demonstrated in the allogeneic neutrophils on day 7 before and after in vitro stimulation.

In conclusion Purified neutrophils isolated from the spleen on day 7 , the early peak of allogeneic transplantation a GVHD, express high levels of IL-21 message and intracellular IL-21.

Keywords: Allogeneic neutrophils; IL-21; Graft-versus-host disease; Transplantation

\section{Abbreviations}

Graft-versus-Host-Disease (GVHD); acute Graft-versus-Host Disease (aGVHD)

\section{Introduction}

We have previously demonstrated in a murine stem cell transplant model, the rise of splenic IL-17 mRNA on day 3, its peak on day 5 and fall by day 7 corresponding to the rise and fall of CD3/CD8 T cells [1]. Day 5 marked the beginning of a larger and more sustained increase in GR-1 myeloid cells. This sequence suggested that the earlier rise in CD3/CD8 T cells secreted signals that stimulated GR-1 related myelopoiesis. Since the neutrophil rise occurred at the approximate peak of acute Graft-Versus-Host Disease (aGVHD), we asked what inflammatory factors could these neutrophils be secreting that might enhance the pathology of GVHD?

Herein we show that purified neutrophils isolated from the spleen on day 7 , the early peak of aGVHD following allogeneic bone marrow transplantation in this donor/host pair, express high levels of IL-21 mRNA and intracellular IL-21.

\section{Materials and Methods}

\section{Animals}

Male BALB/c H2 ${ }^{\mathrm{d}}$ and $\mathrm{C} 57 \mathrm{BL} / 6 \mathrm{H} 2^{\mathrm{b}}$ (B6) mice were purchased from Jackson Laboratories (Bar Harbor, ME). After receipt, they were held for 1 or 2 weeks until they were 9-10 weeks of age. B6 mice were chosen for their MHC incompatibility with BALB/c mice. BALB/C and B6 healthy 9-10 week old males were used as transplant donors. Prior to and after radiation the animals were housed 4 to a cage in micro-isolator cages under the supervision of licensed veterinarians. Sentinel mice to detect viral and bacterial infections in the colonies were always present in the rooms with the mice. This study was carried out in strict accordance with the recommendations in the Guide for the Care and Use of Laboratory Animals of the National Institutes of Health. The protocol was approved by the Institutional Animal Care and Use Committee 596, Lexington, Kentucky. Protocol (THO-12011-AF). 


\section{Bone Marrow, T cell isolation and quantification of the total number of $\mathrm{T}$ cells transplanted}

EasySep Mouse T Cell Enrichment Kit (StemCell Technologies (Vancouver, Canada, \#19751) was used to negatively select for $\mathrm{T}$ Cells in freshly prepared spleen cell suspensions. The reagents for purification were added according to the kit instructions. The viability was 99\% (trypan blue).

Flow cytometry was performed on both the isolated T Cells and the bone marrow cells in order to calculate and adjust the number of total T Cells injected into the recipients. Approximately $1 \times 10^{6}$ cells were simultaneously stained with PE-conjugated CD3 (Clone145-2C11, BD Biosciences, San Jose, CA \#553064) and FITC-conjugated CD45 (Clone 30-F11, Invitrogen \#4501) Mabs. Flow cytometric analysis showed that the bone marrow cells and the enriched cells were $7 \%$ and $98 \%$, respectively, positive for CD3/CD45.

\section{Transplantation}

Allogeneic $\mathrm{H}^{\mathrm{b}} \mathrm{B} 6$ or syngeneic BALB/c donor cells were transplanted into 9-10 week old male $\mathrm{H} 2^{\mathrm{d}} \mathrm{BALB} / \mathrm{c}$ hosts that had received 8.5 Gy TBI delivered by a Mark IV 137Cesium irradiator (J.L Shepard, Glendale CA) at a dose rate of $1.65 \mathrm{~Gy} / \mathrm{min}$. The final suspension of donor cells injected into each recipient consisted of $10 \times 10^{6}$ bone marrow cells plus splenic T cells for a total of $1.5 \times 10^{6}$ allogeneic T-cells or $1.5 \times 10^{6}$ syngeneic T cells. The cells were injected via a tail vein and contained in a volume of 0.25 $\mathrm{ml}$ of PBS. Following AAALAC and our local guidelines, animals that met prescribed criteria were euthanized and counted as an experimental death. Each cage contained BALB/c mice transplanted with two each of the allogeneic transplants or syngeneic cells to eliminate a possible confounding "cage effect".

\section{Neutrophil isolation}

After $\mathrm{CO}_{2}$ anesthesia, the mice were then killed by cervical dislocation. The spleens were removed by sterile dissection.

Preparation of spleen cells: After rinsing 3 times in sterile PBS, the spleens were perfused with $1-2 \mathrm{ml}$ of RPMI+10\% FCS, placed in a sterile plastic bag containing $5 \mathrm{ml}$ of media and dissociated with a Stomacher (Model 80, Seward Limited, Norfolk, U.K.) for 30 seconds on low setting. Cells were filtered through a $70 \mu \mathrm{m}$ cell strainer, centrifuged at $550 \mathrm{xg}$ for 7 minutes and adjusted to $1 \times 10^{6}$ cells/ml of RPMI 1640 (Invitrogen, Grand Island, NY) + 10\% FBS (Hyclone, Logan, UT) + 2mM L-Glutamine $+100 \mathrm{units} / \mathrm{ml}$ Penicillin and $100 \mathrm{ug} / \mathrm{ml}$ Streptomycin (Sigma, St. Louis, M0). All operations were performed at room temperature.

Preparation of purified neutrophils: The Stem Cell Technology's EasySep Mouse Neutrophil Enrichment Kit was used to negatively select for neutrophils in freshly prepared spleens. $2 \times 10^{8}$ nucleated cells contained in $2 \mathrm{ml}$ of Phosphate Buffered Saline (PBS) were added according to the kit instructions. The viability was checked with trypan blue and purity was verified by 2-color flow cytometry with anti-Ly6G FITC (Clone 1A8, BD Biosciences, San Jose, CA, \#551460) and anti-CD11 $\beta$ PE (Clone M1/70, BD Biosciences, \#559911) and hamster anti mouse
CD3 (Life Technologies, Grand Island NY). Appropriate isotype controls were also run.

\section{RNA isolation, cDNA synthesis and real-time PCR}

Total RNA isolation: An aliquot of the isolated neutrophils was finely minced and homogenized (Tissue Tearor, Biospec Products, Inc. Bartlesville, OK) at RT in RLT buffer (RNeasy isolation kit Qiagen Inc, Valencia, CA) + B-mercaptoethanol (Sigma-Aldrich, St. Louis, MO). Total RNA was eluted in 54$60 \mathrm{vl}$ of diethyl procarbonate (DEPC) $\mathrm{H}_{2} \mathrm{O}$. Quantitation ( $\mu \mathrm{g} /$ $\mathrm{ml}$ ) and purity (260/280 ratio) was assessed by use of a spectrophotometer and RNA quality was analyzed on an Agilent Bioanalyzer.

cDNA synthesis: $200 \mu \mathrm{g}$ of total RNA was used for each $20 \mu \mathrm{l}$ cDNA synthesis reaction. Reagents were purchased from Invitrogen Corporation (Carlesbad, CA); Temperatures were used for synthesis reactions according to protocol. Each $20 \mu \mathrm{l}$ cDNA reaction was then diluted 1:5 for use as template for RealTime PCR reactions.

Real-Time PCR Amplification: $4 \mu \mathrm{l}$ of cDNA was used as template for each $20 \mu \mathrm{l}$ PCR reaction. GAPDH primers were used as an endogenous control. All reagents and equipment were purchased from Applied Biosystems: TaqMan Fast Universal PCR Master Mix (2X) and TaqMan Gene Expression Assays for IL-21, GM-CSF, IFN $\gamma$, TNF $\alpha$, IL-12 (p40) G-CSF, IL-6, and IL-22 were utilized in the 7500 Fast Real-Time PCR System.

Real-Time PCR Analysis: $\Delta \Delta \mathrm{Ct}$ analysis was performed as follows:

1) Duplicate/triplicate Ct values were obtained. After subtracting the GAPDH average endogenous gene expression, the fold difference between the gene expressions in the experimental sample was compared to the control sample (a pool of completely normal BALB/c mice).

\section{Intracellular cytokine}

For the intracellular cytokine analysis, an aliquot of the purified neutrophils was washed once in cold D-PBS. The pellet was resuspended in RPMI+10\% FCS (supplemented with $2 \mathrm{mM}$ Glutamine and $100 \mathrm{U} / \mathrm{ml}$ Penicillin $+100 \mathrm{ug} / \mathrm{ml}$ Streptomycin), aliquoted into $5 \mathrm{ml}$ polypropylene tubes @2 x 10 $0^{6} \mathrm{cells} / \mathrm{ml}$. Cells were then treated either with Cell Stimulation Cocktail (ionomycin plus phorbol) plus protein transport inhibitor eBioscience, San Diego, CA) or with just the protein transport inhibitor alone and incubated for 6 hours inside a $5 \% \mathrm{CO}_{2}$ incubator at $37^{\circ} \mathrm{C}$. After the incubation, the cells were centrifuged, washed $2 \mathrm{X}$ in cold D-PBS+1\% FCS (Staining Buffer), blocked with CD16/32, and then stained on ice with GR-1 PE, F4/80 FITC, or CD45 FITC cell surface Moabs (Life Technologies, Grand Island, NY). After washing $2 \mathrm{X}$ with Staining Buffer, cells were then intracellularly stained with Anti-Mouse IL-21 APC Moab or its Isotype Control using an Intracellular Fixation and Permeabilization Kit according to the kit instructions (eBioscience, San Diego, CA). All amounts used for staining either surface marker or intracellular IL-21, inhibition of protein, or cell activation were those recommended 
by the companies. Cell pellets were then suspended in $0.2 \mathrm{ml}$ Staining Buffer and analyzed on a FACSCalibur Flow Cytometer (BD Biosciences, San Jose, CA) using CellQuest Pro software.

\section{Statistical analysis}

Mean mRNA levels were compared by using an analysis of variance for a 3 x 10 factorial design with factors at three levels. Post hoc comparison of means emphasized a comparison of the donor groups on the day of sacrifice provided the interaction between day of sacrifice and donor was significant at the $p<0.05$.

\section{Results}

BALB/c mice transplanted with allogeneic WT B6 BM plus added splenic T lymphocytes develop acute GVHD

Transplantation of allogeneic bone marrow plus added splenic T cells results in acute GVHD (Figure 1). As the weight curve indicates, this is evident by day 5 and weight loss reaches a nadir by day 7. In other studies some of these animals will die during the day 5-10 periods with severe bowel injury but others will survive and gradually regain some of their lost weight. However, by day 14 weight loss begins to recur, signs of chronic GVHD in the skin appear and diarrhea may also begin to become severe by day 21. Many of these animals will die. To the contrary, the mice transplanted with syngeneic cells only lose a little weight by day 3 secondary to total body radiation. They regain much of their weight and, by day 10 , maintain nearly normal weights thereafter and all survive. This figure is shown to serve as the correlative structure for the time points chosen to obtain samples for analysis.

There is a consistent rise in CD3/CD8/CD4 $\mathrm{T}$ cells at day 5 followed by a consistent rise in GR-1 cells by day 7 in the spleens of BALB/c mice transplanted with B6 cells

On days 3, 5, 7, 14, 21 and 31 groups of 5-6 mice in each group were euthanized for analysis of the phenotypes of the cells in their spleen. Figure 2 presents the flow data from BALB/c spleens of the mice transplanted with cells from the allogeneic donors as compared to that from the syngeneic donors. Whereas the total number of nucleated cells in the spleens of the mice transplanted with allogeneic cells rose above the day 3 nadir, it never returned to the pre-transplant baseline (top left panel). To the contrary, the total number of nucleated cells rose in the syngeneic transplants above the normal control baseline on day 14 post transplant before gradually declining by day 31 . The top right panel illustrates that CD19 B cells dropped in all of the B6 transplants to very few cells and never recovered during the 31days. Total CD19 B cells rose dramatically in the syngeneic transplants between day 7 and 14 to levels even greater than that of the normal controls. The middle 2 panels illustrate a significant sharp rise in CD8 and to a lesser extent CD4 T lymphocytes peaking on day 5 . However, note that by day 7 the number of CD8 cells had fallen back almost 4 fold toward baseline and the CD4 cells 2 fold in the spleens from the B6 donors. This sharp transient increase did not occur in the syngeneic transplants. The bottom frame illustrates the pattern of GR-1 reconstitution in the spleen. In contrast, a sharp peak in GR-1 cells arose on day 7 in the spleen cells from the allogeneic transplants at the time that the number of CD8 and CD4 T cells had significantly decreased. The peak of GR-1 cells in the syngeneic transplants occurred on day 14 corresponding to the peak in total cells in the syngeneic transplanted spleens.

In the allogeneic transplants, the spleen was essentially repopulated with $\mathrm{H}-2^{\mathrm{b}}+$ donor cells by day 7 : day $3,32.5+/-$ $24.02 \%$; day 5, $98.71+/-0.15 \%$; day 7, $99.78+/-0.11 \%$.

On day 7 post transplant, B6 allogeneic highly purified GR-1 splenic neutrophils express high levels of IL-21 and significant levels of GM-CSF whereas syngeneic neutrophils express significant levels of TNF $\alpha$

As illustrated in Figure 2, there were two distinct peaks in the GR- 1 cells corresponding to the allogeneic peak on day 7 and the syngeneic peak on day 14 . We asked were their differences in the cytokine expression on these two days? Therefore, neutrophils

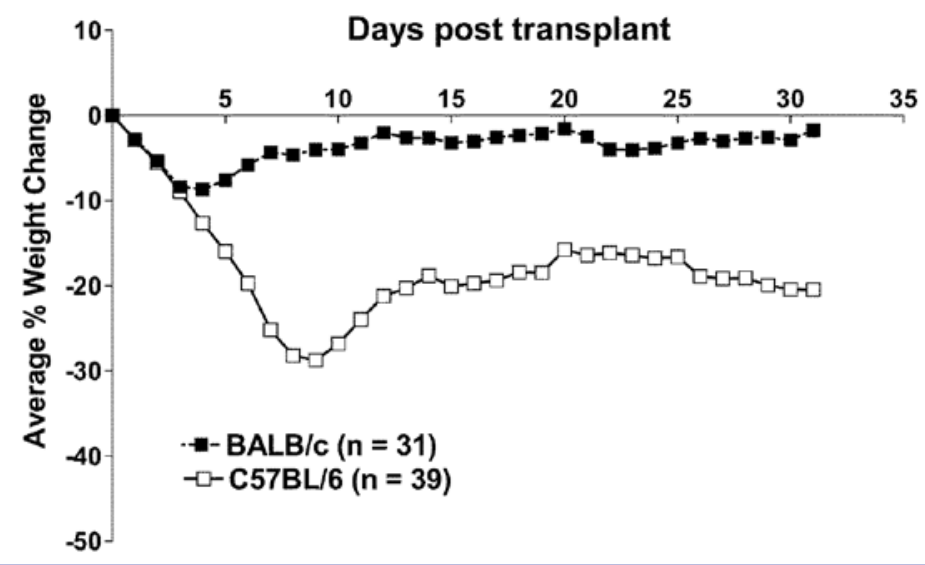

Figure 1: Body weights from 3 consolidated experiments.

$\mathrm{H}-2^{\mathrm{d}}$ BALB/c mice transplanted with cells from $\mathrm{H}-2^{\mathrm{b}} \mathrm{B} 6$ allogeneic or syngeneic BALB/c donors. Each point represents the mean of all of the live animals in their transplant groups weighed on that day. 

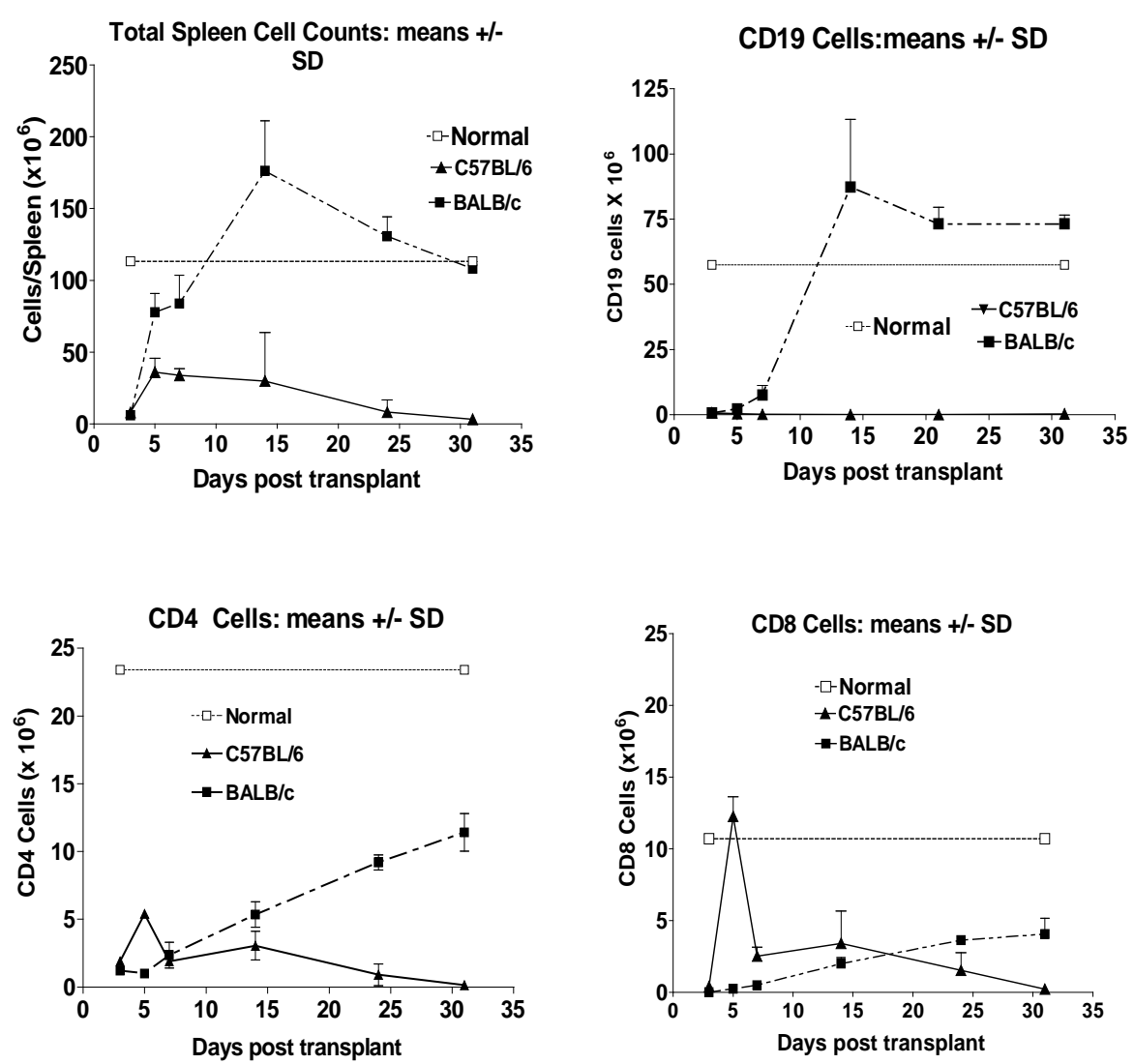

GR-1 Cells: means +/- SD

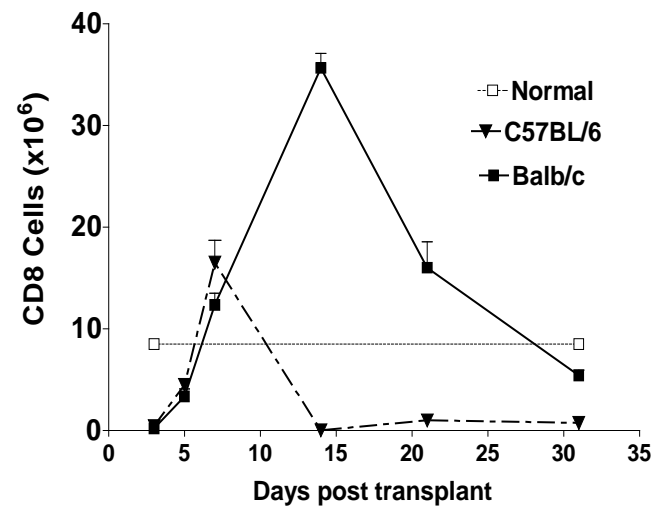

Figure 2: Flow cytometry demonstration of the time and height of phenotypic cells in the spleen of BALB/c mice transplanted with allogeneic and syngeneic cells.

Time and the absolute number of total, CD4, CD8, CD19, and GR-1 cells obtained from the spleens of BALB/c mice transplanted with B6 or syngeneic BALB/c cells on days 3, 5, 7, 14, 21 and 31 days post transplant: Total spleen cells (upper left panel), CD19 B lymphocytes (upper right panel), CD4 and CD8 T lymphocytes (left and right middle panels) and GR-1 cells (lower single panel). The dashed horizontal line in each panel represents the mean of the absolute number of the cells of that phenotype obtained from 6 completely normal BALB/c animals. Means +/- SD $(n=4-6)$.

were isolated from the spleens of BALB/c mice transplanted with allogeneic and syngeneic donor cells on these two days. Purification with LY6G antibody isolates highly purified GR-1 neutrophils and does not include GR-1 macrophages. Figure 3 presents the step wise purification of two completely independent experiments. The resulting number of cells at each step is compared to that from completely normal BALB/c animals. It illustrates two points. First, although the total number of cells in the spleens of the B6 transplanted animals was much less than that from the syngeneic transplants, and second, ,the number of GR-1 cells was greater in the allogeneic on day 7 from that of the 7 day syngeneic and control spleens. 


\section{Figure 3a: Experiment \#1}

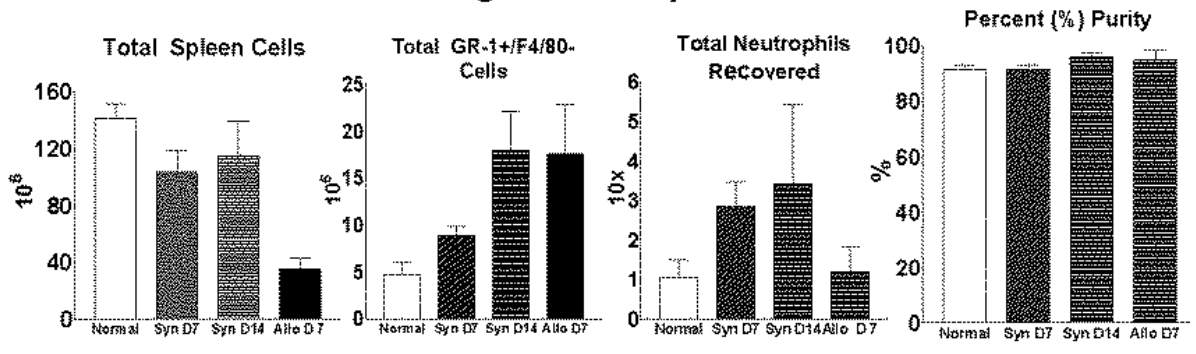

Figure 3b:Experiment \#2

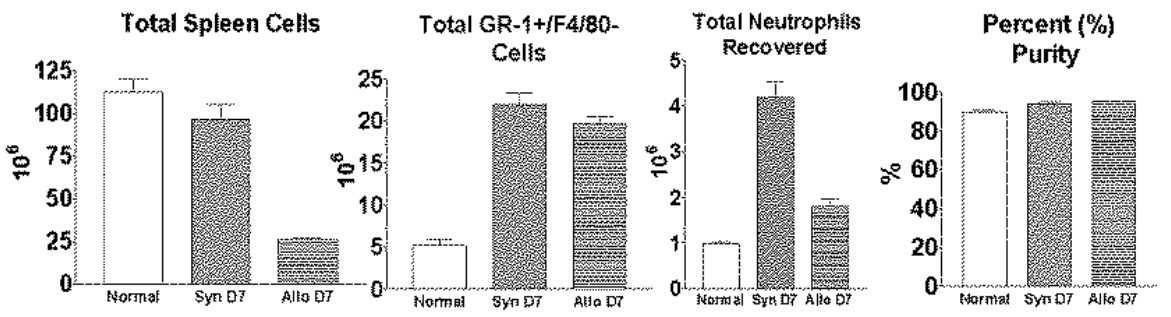

Figure 3: Step-wise isolation of purified neutrophils.

The isolation steps from the spleens of allogeneic (Allo) and syngeneic (Syn) transplants are presented beginning with the total number of cells isolated from the spleen in the upper panel to the percentage of recovery of neutrophils and the purity of each sample on the bottom frames. For comparison the step-wise isolation from the spleens of completely normal BALB/c mice is included but not compared in the bar graphs illustrated in each panel. Means +/- SD. Experiment \#1 (allogeneic day $7 n=5$ ), syngeneic day $7 n=4$, syngeneic day $14, n=5$ ). Experiment \#2, both allogeneic and syngeneic day $7(\mathrm{n}=6)$.

The purification of GR-1 neutrophils from the spleens of mice transplanted with allogeneic B6 cells was quite adequate for analysis on day 7. However, there was a major decrease in the number of allogeneic cells available for purification on day 14 (see Figure 3a) and it was not possible to obtain a sufficient number of highly purified neutrophils for analysis on that day. Therefore, a day 14 analysis was not attempted in the second experiment (Figure 3b). In this experiment we tested specifically for residual CD3 $\mathrm{T}$ cells. The final total number of CD3 $+\mathrm{T}$ cells in the allogeneic neutrophil isolate was $0.0044+/-0.0011 \times 10^{6}$ and in the syngeneic isolate was $0.0008+/-0.0007 \times 10^{6}$. Based on this data, we felt confident that the mRNA expressions and intracellular cytokines observed were not coming from activated T cells.

\section{mRNA expression in purified neutrophils}

In the first experiment (Figure 3a) highly purified (95.0 +/- $3.24 \%$ ) GR-1 positive cells isolated 7 days after allogeneic transplantation expressed high levels of IL-21. The very small number of CD3 + T cells in the both the allogeneic and syngeneic samples strongly supports our conclusion that they were not the source of the high IL-21 expression.

GM-CSF was significantly expressed in the allogeneic but not the syngeneic transplants on day 7 in the first experiment but the level was much lower in the second. Similarly IFN $\gamma$ was significantly increased in both experiments as compared to that in the syngeneic neutrophils on day 7 but the expression level were just above that in the untreated control cells.
This pattern of allogeneic greater than syngeneic expression was reversed with respect to the expression of TNF $\alpha$ which modestly but significantly increased above that in the syngeneic transplants and in the untreated controls. Whereas the levels of IL-12p40 were significantly greater in the syngeneic than the allogeneic neutrophils, the level was less than that in the untreated controls. The expressions of IL-6, G-CSF and IL-22 on day 7 in the allogeneic were not statistically different from that in the syngeneic transplants on day 7.

In the second experiment (see Figure $4 \mathrm{~b}$ ) the purified neutrophils ( $94.8+/-1.53)$ again expressed high levels of IL21 (Figure $4 \mathrm{~b}$ ). As noted above, GM-CSF and IFN $\gamma$ expressions were significantly greater in the allogeneic as compared to the syngeneic transplants but the expressions were just at or below that in the untreated BALB/c controls. Taken together the experiments demonstrate that not only do the syngeneic neutrophils peak 7 days later than that of the allogeneic neutrophils but their expression of cytokines is strikingly different than that in the allogeneic transplants.

\section{Intracellular IL-21}

The next question was did these highly purified neutrophils produce IL-21 protein? Unfortunately, IL-21 has only occasionally been detected in the serum of patients and experimental animals [2], even when there was clear evidence of the critical role this cytokine was playing in the pathogenesis of the clinical or the experimental models. Therefore, we elected 
Figure 4: mRNA expression in isolated splenic neutrophils

Fig. 4a: Experiment Number 1 : means + - SD
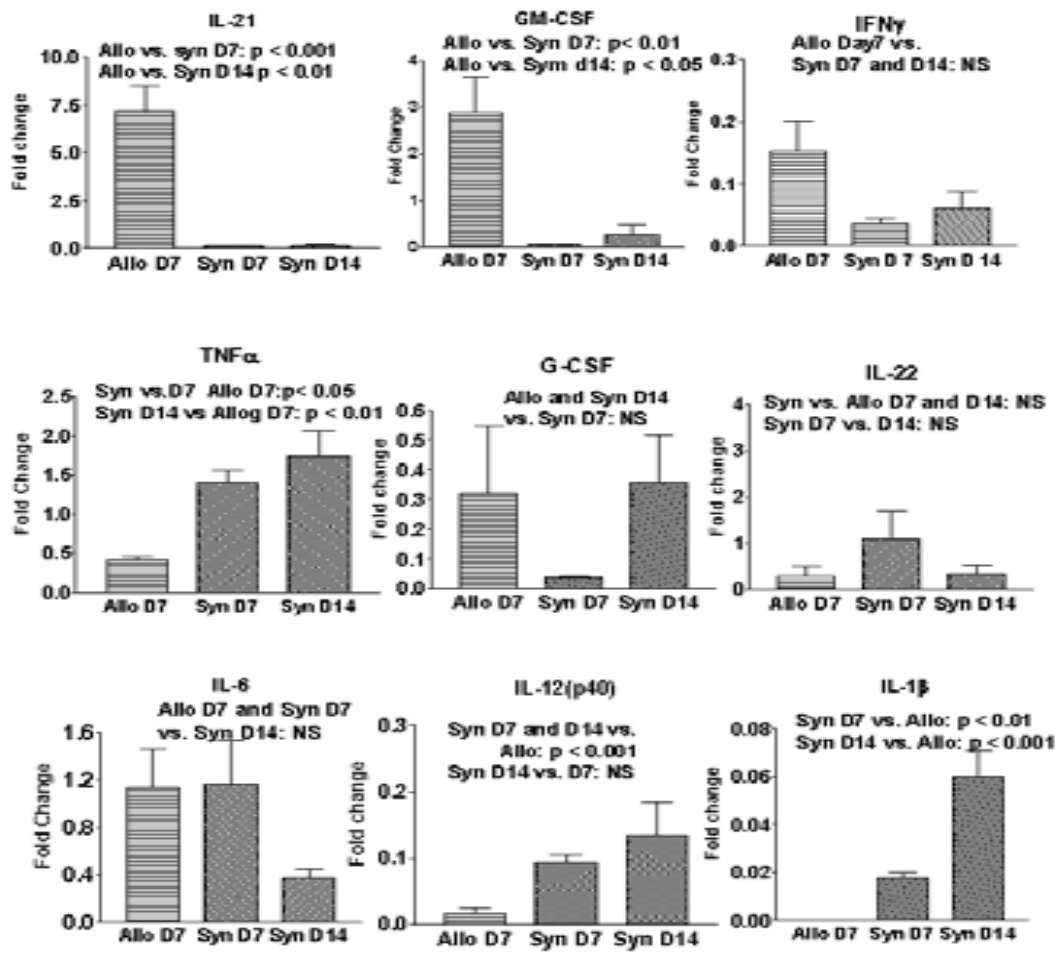

Fig, 4b: Experiment Number 2: means +f- SD
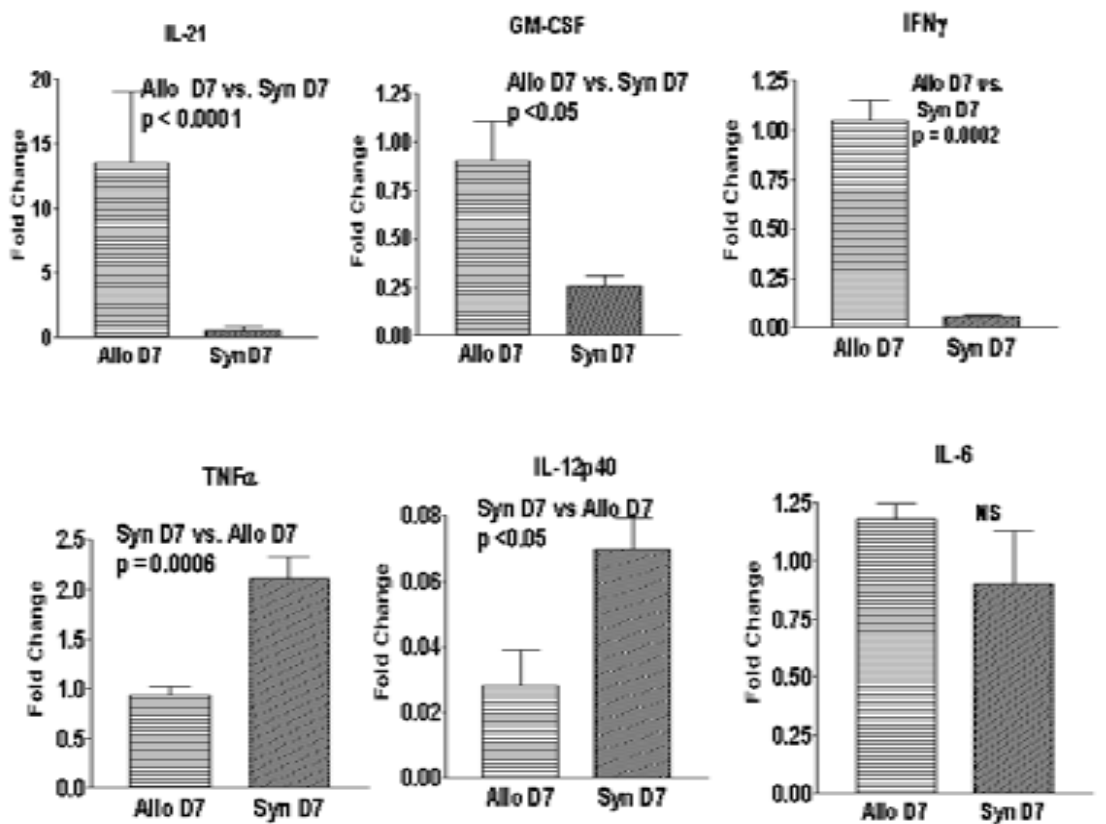

Figure 4: The cytokine expressions of IL-21, GM-CSF, IFN $\gamma$, TNF $\alpha$, IL-12p40 ( $\beta$ ), IL-22, G-CSF and IL-6 in neutrophils isolated from BALB/c mice transplanted with syngeneic (Syn) BALB/c cells (tangential hatched bars to the left, day 7; tangential hatched bars to the right day14) or Allogeneic (Allo) B6 cells (horizontal hatched bars day 7). Note the fold change scale differs with each cytokine: Means $+/-$ SD. Experiment \#1 (allogeneic day 7, $\mathrm{n}=5$ ); syngeneic day $7 \mathrm{n}=4$; syngeneic day $14, \mathrm{n}=5$ ).

Experiment \# 2. Both allogeneic (Allo) and syngeneic (Syn) cells on day 7 ( $\mathrm{n}=6)$.

Tangentially hatched, syngeneic day 7; horizontally hatched, allogeneic day 7. NS = not statistically significant.

Citation: Thompson JS, Hardin DL, Glass JF, Dziba J, Campion J, et al. (2016) The Inflammatory Cytokine IL-21 is Expressed by Splenic Neutrophils in Response to Transplantation of Allogeneic Cells. SOJ Immunol 4(1): 1-9. DOI: http://dx.doi.org/10.15226/2372- 
to directly determine its intracellular presence in the highly purified splenic neutrophils obtained 7days after allogeneic transplantation. Figure 5 illustrates the presence of intracellular IL-21 in these isolated neutrophils before and 6 hours after additional stimulation. Although the difference is relatively small, it is statistically significant. Furthermore, the extremely small number of CD3 positive cells in this preparation $(0.0044$ $+/-0.0011 \times 10^{6}$ ) could not account for even this change.

\section{Discussion}

Neutrophils are the earliest responders in innate immunity [3]. Although they have been traditionally considered as being critical for the elimination of toxins, bacteria, other organisms and foreign bodies [4-6], they also have important inflammatory roles in thermal injury [7], ischemia reperfusion [8], a number of immune related disorders [9-10] and hyperacute solid organ transplant rejection [11]. Depending on the experimental or clinical situation, neutrophils have been reported to secrete IL-8 [12], IL-18 [13], IL-17 [2,6-8] and IFN $\gamma$ [14]. Although, per cell, neutrophils produce much less cytokine protein than monocytes or lymphocytes [2,15], they are more numerous and may be a major cell identified in inflammatory reactions. For example, neutrophils are prominent in sclerosing cholangitis and biliary duct inflammation of the liver $[16,17]$. With respect to GVHD, extensive neutrophil infiltration has been observed in pathology of the colon $[18,19]$ and in the lung [20]. Furthermore, Schwab, et al. [21] demonstrated that physical or genetic depletion of neutrophils improved survival in a mouse model of GVHD.

In our model of BALB/c hosts transplanted with fully allogeneic B6 cells, there is an initial marked loss in total cellularity associated with nearly complete loss of lymphoid tissue in the spleen. This is followed by a new increase in spleen weight and cellularity beginning on day 5 post transplant. It is during this period that neutrophils comprise the major splenic cell population. The striking increase in neutrophils recorded in the allogeneic transplants on day 7 corresponded to the maximum weight loss during acute GVHD. These neutrophils expressed IL-21 and produced IL-21 cytokine. Although the question of the biologic importance of the splenic neutrophil production of IL-21 cannot be resolved with these experiments, it is during this period that neutrophils comprise the major splenic cell population and in total could account for an important source of this cytokine.

IL-21 is an inflammatory cytokine produced by activated Th17 cells [22,23], follicular helper $\mathrm{T}$ cells $[24,25]$ and by activated NK cells [26]. It is also expressed in Hodgkin's lymphoma [27]. Although it has been identified in neutrophils in association with B lymphocytes in the marginal zone of normal spleens [28], by day 7 post allogeneic transplant, lymphoid follicles in the spleen have been destroyed and B cells are essentially absent.

It may have a major role in the pathogenesis of psoriasis [29-31] and inflammatory bowel disease [32,33]. Distler, et al. [34] observed a 4.7 fold increased expression of IL-21 receptor in the epidermis of the skin of patients with scleroderma as compared to that in normal skin. IL-21 may also be a factor in the pathogenesis of GVHD. Experimentally, survival has been significantly improved with transplantation of IL-21-/- donor cells as compared to WT cells [35] and by blockade of IL-21 [36]. Zhao, et al. [37] concluded that IL-17 and IL-21 may be critically important for the pathogenesis of chronic GVHD.

Although GM-CSF was significantly increased in day 7 allogeneic neutrophils, the expression levels were much less than that of IL-21. To our knowledge GM-CSF has not been implicated in the GVHD reaction but it is important to note that its expression was elevated in the allogeneic but not in the syngeneic neutrophils. It is a cytokine secreted by macrophages, T cells, mast cells, NK cells, endothelial cells and fibroblasts. In general it functions as a white blood cell growth factor. In addition, it has been shown to have roles in cell signaling [38] and in promoting inflammatory dendritic cells [39] and macrophages [40]. Furthermore, it has been implicated in the pathology of lung injury [41] and arthritic pain [42].

Although this study does not establish that neutrophil secretion of IL-21 played a critical role in the pathogenesis of aGVHD, it does document that allogeneic but not syngeneic neutrophils in the spleen express and produce this cytokine. The evidence that strikingly increased IL-21 expression occurred
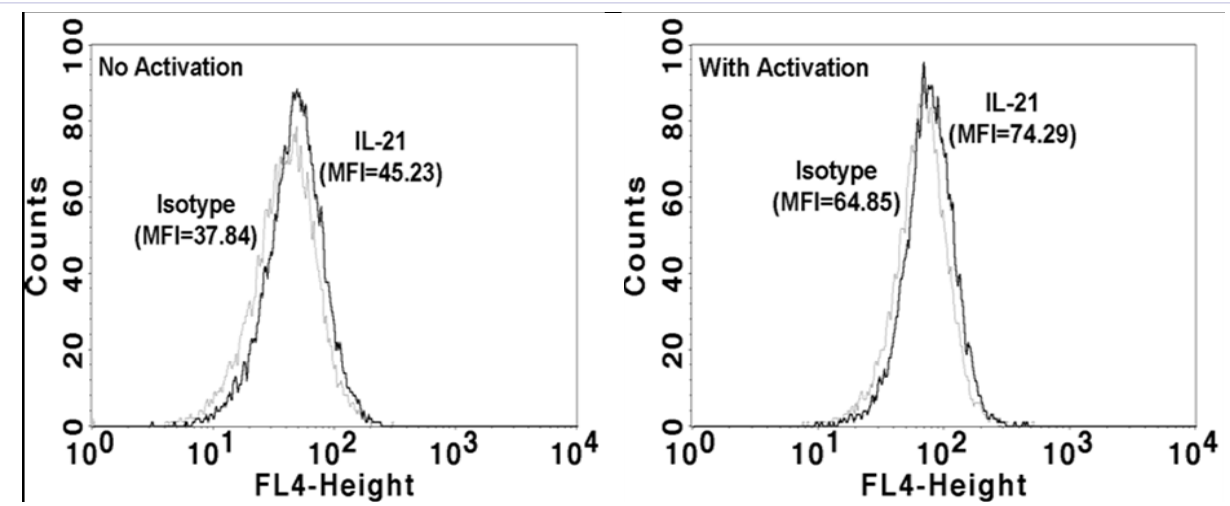

Figure 5: IL-21 intracellular cytokine analysis.

Unstimulated on the left and stimulated with on the right. MFI = Mean Fluorescent Intensity. The solid line is IL-21 and the more faint dotted line is the isotype control. 
only in allogeneic neutrophils suggests that there may be other target organs where neutrophils [18-21] could be producing these cytokines and contributing to their organ pathology.

\section{Conflict of Interest}

The authors declare no conflict of interest.

\section{Support}

Supported by a VA Merit Review Grant

\section{References}

1. Thompson JS, Chu Y, Glass JF, Brown SA. Absence of IL-23/p19 in donor allogeneic cells reduces mortality from graft-versus-host disease. Bone Marrow Transplant. 2010;45(4):712-22. doi: 10.1038/ bmt.2009.215.

2. Tecchio C, Micheletti A, Cassatella MA. Neutrophil-derived cytokines: facts beyond expression. Front Immunol. 2014;21(5):508. doi: 10.3389/fimmu.2014.00508.

3. Jaillon S, Galdiero MR, Del-Prete DD, Cassatella MA, Garlanda C, Mantovani A. Neutrophils in inate and adaptive immunity. Semin Immunopathol. 2013;35(4):377-94. doi: 10.1007/s00281-013-03748.

4. Shibata K, Yamada H, Hara H, Kishihara K, Yoshikai Y. Resident $\mathrm{V}$ delta1+ gamma delta $\mathrm{T}$ cells control early infiltration of neutophils after Escherichia coli infection via IL-17 production. J Immunol.2007;178(7):4466-4472.

5. Miyamoto M, Prause 0 , Sjöstrand M, Laan M, Lötvall J, Lindén A. Endogenous IL-17 as a mediator of neutrophil recruitment caused by endotoxin exposure in mouse airways J Immunol. 2003;170(9):466572 .

6. Werner JL, Gessner MA, Lilly LM, Nelson MP, Metz AE, Horn D, et al. Neutrophils produce IL17 (IL-17A) in a dectin-1-and IL-23dependent manner during invasive fungal infection. Infect Immun. 2011;79(10):3966-77. doi: 10.1128/IAI.05493-11.

7. Chen LW, Huang HL, Lee IT, Hsu CM, Lu PJ. Thermal injury-induced priming effect of neutrophil is TNF- $\alpha$ and p38 dependent. Shock. 2006;26:69-76

8. Li L, Huang L, Vergis AL, Ye H, Bajwa A, Narayan V, et al. IL-17 produced by neutrophils regulates IFN-y-mediated neutrophil migration in mouse kidney ischemia-reperfusion injury. J Clin Invest. 2010;120(1):331-42. doi: 10.1172/JCI38702.

9. Nakagome K, Matsushita S, Nagata M. Neutrophilic inflammation in severe asthma. Int Arch Allergy Immunol. 2012;158 Suppl 1: 96-102. doi: $10.1159 / 000337801$.

10. Lukacs NW, Strieter RM, Chensue SW, Widmer M, Kunkel SL. TNF-a mediates recruitment of neutrophils and eosinophils during airway inflammation. J Immunol. 1995;154(10):5411-7.

11. Miura M, El-Sawy T, Fairchild RI. Neutrophils mediate parenchymal tissue necrosis and accelerate the rejection of complete major histocompatibility complex-disparate cardiac allografts in the absence of interferon-gamma. Am J Pathol 2003;162:509-519.

12. Montemurro P, Mariggiò MA, Barbuti G, Cassano A, Vincenti A, Serio G, et al. Increase in Interleukin-8 production from circulating neutrophils upon antibiotic therapy in cystic fibrosis. J Cyst Fibrosis. 2012;11:518-24.
13. Sporriz R, Joller N, Hibi H, Oxenus A. A novel role for neutrophils as critical activators of NK cells. J Immunol. 2008;181(10):7121-30.

14. Ellis TN, Bearman BL. Muirine polymorhonuclear neutrophils produce interferon-g in response to pulmonary infections with Nocardia asteroids. J Leukoc Biol. 2002;72(2):373-81.

15.Scapini P, Calzetti F, Cassatella MA. On the detection of neutrophilderived vascular endothelial growth factor (VEGF). J Immunol Methods. 1999;232(1-2):121-9.

16. C Barbatis, P Grases, H A Shepherd, R W Chapman, J Trowell, D P Jewell, et al. Histological features of sclerosing cholangitis in patients with chronic ulcerative colitis. J Clin Path. 1985;38:778-784.

17. Licata LA, Nguyen CT, Burga RA, Falanga V, Espat NJ, Ayala A, et al. Biliary obstruction results in PD-1-dependent liver $\mathrm{T}$ cell dysfunction and acute inflammation mediated by Th17 cells and neutrophils. J Leukoc Biol. 2013;94(4):813-23. doi: 10.1189/jlb.0313137.

18. Eigenbrodt ML, EigenbrodtEH, Thiele D. Histologic similarity of murine colonic graft-versus-host disease (GVHD) to human colonic GVHD and inflammatory bowel disease. Am J Pathol. 1990;137(5):1065-76.

19. Socié G, Mary JY, Lemann M, Daneshpouy M, Guardiola P, Meignin V, et al. Prognostic value of apoptotic cells and infiltrating neutrophils in graft-versus-host disease of the gastrointestinal tract in humans. Blood. 2004;103(1):50-57.

20. Gowdy KM, Nugent JL, Martinu T, Potts E, Snyder LD, Foster WM, et al. Protective role of T-bet and Th1 cytokines in pulmonary graft-versushost disease and peribronchiolar fibrosis. Am J Respir Cell Mol Biol. 2012;46(2):249-56. doi: 10.1165/rcmb.2011-01310C.

21. Schwab L, Goroncy L, Palaniyandi S, Gautam S, Triantafyllopoulou A, Mocsai A, et al. Neutrophil granulocytes recruited upon translocation of intestinal bacteria enhance graft-versus-host disease via tissue damage. Nat Med. 2014;20(6):648-54. doi: 10.1038/nm.3517.

22. Liu SM, King C.IL-21-producing Th cells in immunity and autoimmunity. J Immunol. 2013;191(7):3501-6. doi: 10.4049/jimmunol.1301454.

23. Wei L, Laurence A, Elias KM, O'Shea JJ, et al. IL-21 is produced by Th17 cells and drives IL-17 production in a STAT3-dependent manner. J Biol Chem. 2007;282(48):34605-10.

24.Vogelzang A, McGuire HM, Yu D, Sprent J, Mackay CR, King C. A fundamental role for interleukin-21 in the generation of $\mathrm{T}$ follicular helper cells. Immunity. 2008;29(1):127-37. doi: 10.1016/j. immuni.2008.06.001.

25. Lüthje K, Kallies A, Shimohakamada Y, Belz GT, Light A, Tarlinton DM, et al. The development and fate of follicular helper $\mathrm{T}$ cells defined by an IL-21 reporter mouse. Nat Immunol. 2012;13(5):491-8. doi: $10.1038 /$ ni.2261.

26. Coquet JM, Kyparissoudis K, Pellicci DG, Besra G, Berzins SP, Smyth MJ, et al. IL-21 is produced by NKT cells and modulates NKT cell activations and cytokine production. J Immunol 2007;178:2827-2834.

27.Scheeren FA, Diehl SA, Smit LA, Beaumont T, Naspetti M, Bende $\mathrm{RJ}$, et al. IL-21 is expressed in Hodgkin lymphoma and activates STAT5: evidence that activated STAT5 is required for Hodgkin lymphomagenesis. Blood. 2008;111:4706-4715.

28. Puga I, Cols M, Barra CM, He B, Cassis L, Gentile M, et al. B cellhelper neutrophils stimulate the diversification and production of immunoglobulin in the marginal zone of the spleen. Nat Immun. 2012;13:170-180.

29. Caruso R, Costanzo A, Monteleone G. Pathogenic role of interleukin-21 
in psoriasis. Cell Cycle. 2009;8:3629-3630.

30. He Z, Jin L, Liu ZF, Hu L, Dang EL, Feng ZZ, et al. Elevated serum levels of interleukin 21 are associated with disease severity in patients with psoriasis. Br J Dermatol 2012;167:191-193. doi: 10.1111/j.13652133.2012.10899.x.

31. Botti E, Spallone G, Caruso R, Monteleone G, Chimenti S, Costanzo A Psoriasis, from pathogenesis to therapeutic strategies: IL-21 as a novel potential therapeutic target. Curr Pharm Biotechnol. 2012;13:18611867.

32. Pallone F, Fina D, Caruso R, Monteleone G. Role of IL-21 in inflammatory bowel disease. Expert Rev Clin Immunol. 2010;6(4):537-41. doi: 10.1586/eci.10.44.

33. MacDonald TT, Bell I, Monteleone G. The opposing roles of IL-21 and TGF $\beta 1$ in chronic inflammatory bowel disease. Biochem Soc Trans. 2011;39:1061-1066. doi: 10.1042/BST0391061.

34. Distler JH, Jüngel A, Kowal-Bielecka O, Michel BA, Gay RE, Sprott H, et al. Expression of Interleukin-21 receptor in epidermis from patients with systemic sclerosis. Arth Rheum. 2005;52:856-864.

35. Meguro A, Ozaki K, Oh I, Hatanaka K, Matsu H, Tatara R, et al. IL21 is critical for GVHD in a mouse model. Bone Marrow Transpl. 2010;45:723-729.

36. Bucher C, Koch L, Vogtenhuber C, Goren E, Munger M, PanoskaltsisMortari A, et al. IL-21 blockade reduces graft-versus-host disease mortality by supporting inducible $\mathrm{T}$ regulatory cells generation. Blood. 2009;114:5375-5384.
37.Zhao XY, Lv M, Xu LL, Qian X, Huang XJ, et al. Donor IL-17 cells and IL-21 may contribute to the development of chronic graft-versushost disease after allogeneic transplantation. Eur J Immunol. 2013;43(3):838-50. doi: 10.1002/eji.201242816.

38. Parajuli B, Sonobe Y, Kawanokuchi J, Doi Y, Noda M, Takeuchi H, et al. GM-CSF increases LPS-induced production of proinflammatory mediators via upregulation of TLR4 and CD-14 in murine microglia. J Neuroinflammation. 2012;9:268. doi: 10.1186/1742-2094-9-268.

39. Blyszczuk P, Behnke S, Lüscher TF, Eriksson U, Kania G. GM-CSF promotes inflammatory dendritic cell formation but does not contribute to disease progression in experimental autoimmune myocarditis. Biochim Biophys Acta. 2013;1833(4):934-44. doi: 10.1016/j.bbamcr.2012.10.008.

40. Sorgi CA, Rose S, Court N, Carlos D, Paula-Silva FW, Assis PA, et al. GM-CSF priming drives bone marrow-derived macrophages to a pro-inflammatory pattern and down modulates PGE2 in response to TLR2 ligands. PLoS One. 2012;7(7):e40523. doi: 10.1371/journal. pone.0040523.

41. Choi JC, Jung JW, Kwak HW, Song JH, Jeon EJ, Shin JW, et al. Granulocyte macrophage-colony stimulating factor (GM-CSF) augments acute lung injury via its neutrophil's priming effects. J Korean Med Sci. 2008;23(2):288-95. doi: 10.3346/jkms.2008.23.2.288.

42. Cook AD, Pobjoy J, Sarros S, Steidl S, Dürr M, Lacey DC, et al. Granulocyte-macrophage colony-stimulating factor is a key mediator in inflammatory and arthritic pain. Ann Rheum Dis. 2013;72(2):26570. doi: 10.1136/annrheumdis-2012-201703. 